\title{
INVESTIGACIONES
}

\section{Normas de convivencia escolar: Descripción y análisis de un proceso participativo}

\author{
Rules of school coexistence: \\ Description and analysis of participatory process
}

Alejandra Ávalos Díaz, Christian Berger Silva ${ }^{b}$

${ }^{a}$ Investigadora independiente en el área de convivencia escolar. adavalos@uc.cl

${ }^{b}$ Escuela de Psicología de la Pontificia Universidad Católica de Chile. cberger@uc.cl

\section{RESUMEN}

A pesar de que existe evidencia de que la convivencia escolar mejora cuando las normas son creadas participativamente, se carece de evidencia empírica que muestre cómo realizar estos procedimientos. El presente, es un estudio cualitativo que tiene por objetivo describir y analizar el desarrollo de un proceso participativo de construcción de normas de convivencia escolar, en una escuela básica municipal, el cual contó con el apoyo de una mediadora que intencionó la participación de todos los actores de la comunidad escolar. Los datos se recogieron a partir de focus group con profesores y asistentes de la educación, junto con el análisis del registro de campo llevado por la mediadora durante todo el proceso. Los resultados se analizaron con teoría fundamentada y describen empíricamente el desarrollo de una serie de etapas, junto con identificar factores facilitadores y obstaculizadores, además de los beneficios que generó el proceso participativo en los actores.

Palabras claves: participación, normas de convivencia escolar, formación ciudadana.

\section{ABSTRACT}

Although there is evidence that school coexistence improves when rules are created participatively, there is not empirical evidence showing how to perform these procedures. The present, is a qualitative study that aims to describe and analyze the development of a participatory process of construction of rules of school coexistence, in a public primary school, which had the support of a mediator who intended the participation of all actors of the school community. The data was collected from the focus group with teachers and education assistants, together with the analysis of the field record taken by the mediator during the whole process. The results were analyzed with well-founded theory and empirically describe the development of a series of stages, along with identifying facilitating and impeding factors, as well as the benefits generated by the participatory process in the actors.

Key words: participation, school policy, citizen training. 


\section{INTRODUCCIÓN}

En la actualidad, existe consenso entre docentes e investigadores de que la convivencia escolar registra mejoras significativas cuando se logra involucrar en su gestión a todos los miembros de la comunidad escolar. Para que las normativas y reglamentos de convivencia escolar cumplan la función de, por una parte, facilitar las relaciones cordiales entre los diferentes miembros y, por otra, guíen a la hora de intervenir ante los conflictos que surjan, es necesario que esas normas tengan un sentido para los distintos actores educativos (Torrego, 2006), lo cual es más probable que ocurra si las normas contenidas en ellos son compartidas por todos los miembros de la comunidad escolar. En caso contrario -al ser impuestas- genera en los estudiantes una relación externalista con estas, donde es difícil que se llegan a legitimar, generando su acatamiento voluntario (Ruiz y Chaux, 2005).

A nivel legislativo, las exigencias tanto en la mejora de la convivencia como en el aumento en la participación al interior de las comunidades educativas en nuestro país son cada vez mayores. En el año 2005, se establece la obligatoriedad de los concejos escolares en todos los establecimientos que reciben subvención del Estado, que cuente con la participación de representantes de todos los estamentos de la escuela. Así también, la ley SEP prescribe el diseño de planes de mejora, para invertir los recursos asignados, los cuales deben ser realizados con la participación de la comunidad, principalmente apoderados y estudiantes. En 2011 se promulga la ley sobre violencia escolar que entrega a los alumnos, alumnas, padres, madres, apoderados, profesionales y asistentes de la educación, así como los equipos docentes y directivos de los establecimientos educacionales la responsabilidad de propiciar un clima escolar que promueva la buena convivencia de manera de prevenir todo tipo de acoso escolar. Por otra parte, los recientemente incorporados "Otros indicadores de calidad", incluyen evaluaciones a diferentes temáticas dentro de las cuales está la participación y formación ciudadana y el clima de convivencia escolar (Ministerio de Educación, Chile, 2014). Por último, la reciente ley que crea los planes de formación ciudadana busca que las escuelas desarrollen planes para formar competencias en sus estudiantes que les permitan vivir y participar de una sociedad democrática (Ministerio de Educación, Chile, 2016).

Si bien, tanto la teoría como la actual legislación reportan los beneficios de que los distintos actores tengan un rol activo en la construcción de normas de convivencia escolar, los escasos estudios que indagan en estos procesos coinciden en señalar que en general esto no ocurre. Más aún, la literatura da cuenta de los múltiples obstáculos que aparecen cuando se intenta implementar (Rivera y Milicic, 2006; Valdés y Urías, 2011), tanto en la relación entre actores como entre estamentos, lo que finalmente crea un panorama desalentador. Por lo tanto, en la práctica, intentar implicar a la comunidad educativa en la toma de decisiones en la escuela, termina siendo "mera retórica, mera declaración de intenciones, que caminan sigilosamente por los centros y no acaban de convertirse en una práctica cotidianamente asumida" (Bardisa, 1997, p. 34).

Por otra parte, poco sabemos aún sobre los procedimientos empíricos para poder convertir las prácticas participativas en realidad. De hecho, son frecuentes aun en la actualidad las elaboraciones de reglamentos de convivencia "a puerta cerrada", sin explicitar quién lo elabora, cuándo y cómo, generando un desconocimiento generalizado en las comunidades educativas (Litichever y Núñez, 2005; Casas et al., 2008).

Por lo anterior, el objetivo de la presente investigación es describir y analizar el desarrollo de un proceso participativo mediado de construcción de normas de convivencia 
escolar en una escuela municipal. Como en la escuela existía una escasa participación de la comunidad educativa, fue necesario la presencia de la mediadora, que facilitó el diálogo y la creación de consensos entre los participantes. Los objetivos específicos son: a) Identificar y describir los momentos y niveles de participación en un proceso participativo de construcción de normas de convivencia escolar; b) Identificar y describir el rol del mediador en el proceso participativo; y c) Describir los facilitadores y obstaculizadores del proceso participativo de construcción de normas de convivencia escolar.

Conocer más en profundidad sobre el desarrollo de un proceso como este, sus características, sus facilitadores y obstaculizadores ayudará a que más escuelas incorporen prácticas participativas en sus normas de convivencia escolar y en la gestión de la convivencia en general.

\section{CONVIVENCIA ESCOLAR}

Convivir significa "vivir unos con otros sobre la base de unas determinadas relaciones sociales y unos códigos valorativos, forzosamente subjetivos, en el marco de un contexto social determinado" (Jares, 2006, p. 468).

Para entender la complejidad de este fenómeno, analizaremos la clasificación que realiza Carbajal (2013) quien distingue entre una visión reducida y una visión amplia para entender la convivencia escolar.

En la visión reducida, las estrategias de abordaje de la convivencia escolar se centran en el control de comportamientos agresivos de los niños, la cual a su vez refuerza la idea de que los estudiantes, como individuos, son responsables de la violencia en las escuelas (Carbajal, 2013). Existe abundante evidencia tanto a nivel nacional como internacional de que mejorar la convivencia escolar es una estrategia eficaz en la evitación de la violencia en las escuelas (Ortega et al., 2013). En esta línea, buena parte de las políticas de prevención de violencia escolar utilizan esta lógica.

Desde la visión reducida se espera que los conflictos desaparezcan de las escuelas, por considerarse aberrantes y patológicos, como si fueran una enfermedad que invade al cuerpo de la organización (Ball, 1994), además de estar relacionados con la violencia y la indisciplina (Jares, 2006), lo que se enmarca dentro de un paradigma tecnócrata-positivista, que considera deseable su eliminación (Jares, 1997). En este intento, muchas veces los grupos terminan evadiendo su abordaje, lo que genera simplemente que el conflicto no se resuelva, o peor aún, se agudice.

En una visión amplia de convivencia escolar, se integran las relaciones democráticas y las estructuras de participación como elementos orientados a la construcción y consolidación de la paz, entendiendo esta última no sólo como la ausencia de violencia, sino que, como relaciones humanas basadas en la justicia social, la equidad y la autorrealización. Por lo tanto, la visión amplia permea todo el currículum, ya que se orienta a la transformación de las prácticas tanto en el aula como en la escuela (Carbajal, 2013).

En la visión amplia, el rol del conflicto cambia, ya que aborda las raíces estructurales de este en busca de relaciones justas y democráticas que contribuyan al bienestar de la sociedad en general (Carbajal, 2013). Revalorizar el conflicto y considerarlo necesario y útil para el cambio, el aprendizaje y la mejora del sistema educativo, es entenderlo desde un paradigma crítico, lo que favorece su afrontamiento, además de favorecer los 
procesos colaborativos de la gestión escolar (Jares, 1997). Para ello, es necesario su abordaje en instancias tanto formales, como informales, siendo importante incorporar en el establecimiento herramientas formativas y de gestión que permitan ver los conflictos como oportunidad y no como amenaza (Mena et al., 2011).

\section{LOS REGLAMENTOS DE CONVIVENCIA ESCOLAR}

El Reglamento de Convivencia es un documento oficial de los establecimientos educativos en el cual:

se ha definido un conjunto de normas que orientan las relaciones interpersonales entre los distintos actores y permiten lograr las metas institucionales. Regula el comportamiento de los distintos actores de acuerdo al rol que tienen dentro de la comunidad educativa en los distintos ámbitos de la vida escolar, en función del logro de los objetivos de aprendizaje (Bugueño y Mena, 2008, pp. 1-2).

En Chile, la Ley sobre violencia escolar 20.536/2011 exige que cada establecimiento deba contar con un reglamento que regule las relaciones al interior del establecimiento y que en materia de convivencia escolar incorpore políticas de prevención, medidas pedagógicas y protocolos de actuación, además de estipular faltas, graduándolas de acuerdo a su menor o mayor gravedad, estableciendo medidas disciplinarias ante ellas. El establecimiento de normas y reglamentos no basta para el desarrollo de la convivencia escolar, más bien responde a la conformación de un ambiente organizado que facilita su aprendizaje (Ministerio de Educación, Chile, 2015).

Desde una visión reducida de la convivencia escolar, tanto los reglamentos como las normas de convivencia contenidas en estos, nacen de la necesidad de regular y abordar la existencia de conflictos y violencia al interior de las escuelas (Torrego, 2006). Esto, debido a que cuando las normas son arbitrarias, elaboradas al margen de la participación del alumnado, inconsistentes y poco claras, la violencia tiene altas posibilidades de aparecer (Ortega, 1998).

Desde una visión amplia de convivencia escolar, el reglamento debiera establecer algo más que reglas de disciplina, entregando pautas de relación tanto entre las personas como entre los diferentes estamentos de la escuela. Estas pautas debieran ser cada vez más democráticas, para lo cual es necesaria la participación de todos los estamentos tanto en su elaboración como en su revisión (Casas et al., 2008). Este paso no es sencillo, considerando que a las escuelas no se les ha preparado para ello y que no se dispone de suficientes prácticas documentadas que orienten en esta dirección. El problema de esto es que la aparente desestructuración de prácticas participativas termina produciendo frustración y desgaste en las comunidades, lo que las hace, muchas veces, volver a retomar viejos estilos autoritarios (Sús, 2005).

\section{LA PARTICIPACIÓN EN LA ESCUELA}

Si bien son muchas las publicaciones que destacan la importancia de la participación en la sociedad, pocos de ellos se detienen en definir este concepto. En el ámbito de la 
gestión escolar Torrego (2006) señala que participar es "Implicar a todos los miembros de la comunidad educativa a través de la palabra y la acción cooperativa, es decir, el diálogo y la realización conjunta de proyectos" (p. 147). Sin embargo, involucrar a los actores en acciones cooperativas no es un asunto sencillo, ya que en la institución escolar encontramos, por una parte, diversos grupos organizados de modo jerárquico, y por otra, los ideales de igualdad y democracia, donde estos modos de organización, que parecen más bien antagónicos, no tienen equivalentes en otra institución y "se ven obligadas a coexistir en la misma estrecha proximidad" (Ball, 1994, p. 12).

En cuanto a los estudiantes, cuyo proceso formativo es el centro del sistema educativo, su participación implica aún más complejidades. Se entiende por participación infantil "los procesos de compartir las decisiones que afectan la vida propia y la vida de la comunidad en la cual se vive" (Hart, 1993, p. 5). Sin embargo, el paso de reconocer la titularidad de niños y niñas en el ejercicio de sus derechos, como lo establece la Convención Internacional de los Derechos de los Niños, más que como objeto de beneficencia o caridad adulta, resulta aún controvertido y desafiante (Mundaca y Flores, 2014). Si bien se ve con frecuencia la participación de los niños de manera decorativa o simbólica dentro de las instituciones, rara vez son considerados en la toma de decisiones, que es lo que define una participación genuina (Hart, 1993).

La participación en la construcción y significación de las normas en la vida pública constituyen, además, el eje de la formación ciudadana, debido a que si entendemos la escuela como un microsistema social, con una estructura social y política, una forma de funcionamiento y un sistema de justicia, aquello que se aprende en cuanto a valores, resolución de conflictos y estilos de relaciones, serán repetidos por los futuros ciudadanos en las posiciones que ocupen en la sociedad cuando sean adultos (Arón y Milicic, 2000). Ruiz y Chaux (2005) plantean que la ciudadanía se puede acatar o ejercer. "Ejercer la ciudadanía en la escuela significa que sus actores, esto es, los estudiantes, padres de familia, directivos, docentes y trabajadores, participen activamente en la regulación de la vida social en el contexto escolar" (p. 17). Resaltan la importancia de ejercer la ciudadanía como forma de transformar y mejorar las instituciones, sentenciando que "si una persona o grupos de personas no pueden participar en la crítica a las instituciones sociales y en su mejoramiento, incluyéndose aquí la escuela misma, no puede hablarse propiamente de una educación ciudadana" (p. 19).

\section{MOMENTOS Y NIVELES DE PARTICIPACIÓN}

Es necesario especificar que la participación en el ámbito escolar se puede dar de variadas formas, en distintos niveles y en diferentes momentos de un proceso.

Dentro de un proceso participativo Torrego (2006) describe cinco momentos o fases en los que se puede producir la participación: a) en la fase de información, consulta o sensibilización en el tema, donde es imprescindible una buena comunicación partiendo de las necesidades de todos; b) en el proceso de debate, donde se creen canales adecuados para la discusión abierta entre todas las partes; c) en la fase de toma de decisiones, donde radica el principio fundamental de la participación y donde es necesario que todos perciban real poder compartido; d) en la ejecución de esas decisiones, en el cual cada miembro asuma compromisos contraídos conjuntamente y e) en el seguimiento y evaluación, donde se analizan las dificultades en todo el proceso y se pueden hacer continuas propuestas de mejora. 
En cuanto a los niveles de participación en la escuela, se han descrito los siguientes: a) información, en el cual los participantes pueden formarse una opinión en relación a los diversos temas, así como también conocer los alcances y restricciones de su participación; b) colaboración, ejemplo de ello es cuando las escuelas abren espacios para que las familias brinden su apoyo en actividades, ya sea presenciales (como asistencia a actos o eventos), pedagógicos (apoyo en tareas) o financiero (gestionar fondos); c) consulta, donde se pretende recabar opiniones que servirán de insumos al sistema escolar para la planificación, diseño, ejecución y evaluación de acciones a nivel de convivencia escolar, gestión pedagógica, etc., sin embargo, esta consulta puede ser o no considerada por la institución al momento de resolver estos asuntos; d) toma de decisiones, que implica incorporar a representantes con derecho a voz y voto, y cuyo principal beneficio, además de ser una real instancia de participación, es que valida las decisiones tomadas frente a toda la comunidad educativa; y e) control de eficacia, en el cual las familias y la comunidad realizan supervisión del cumplimiento del proyecto educativo y la gestión del establecimiento, el cual sólo es posible de conseguir si se han cumplido con todos los niveles anteriores y se ha generado una cultura de participación (Flamey et al., 1999; Gubbins y Berger, 2002; Romagnoli y Gallardo, 2007).

El nivel de toma de decisiones, que implica la validación de las medidas tomadas por parte de la comunidad educativa, encierra complejidades que no siempre son tan sencillas de percibir. "La toma de decisiones no es un proceso racional abstracto que pueda ser expuesto en un gráfico organizativo; es un proceso político, es la sustancia de la actividad micropolítica" (Ball, 1994, p. 17). Entender las escuelas como lugares políticos, implica entender que al interior de ellos existen personas y grupos con diferentes intereses que "emergen cuando los individuos se dan cuenta de que tienen objetivos comunes y de que pueden ser capaces de ejercer colectivamente alguna influencia para incidir en una decisión que de modo individual no alcanzarían" (Bardisa, 1997, p. 23).

\section{MÉTODO}

\subsection{DISEÑO}

El presente estudio utiliza como método el estudio de caso, de tipo intrínseco, el cual busca estudiar en profundidad un caso en particular para conocer la complejidad de este, más que generar representatividad o generalizaciones a otros casos (Stake, 1994). La elección del caso se realizó por el fácil acceso a este durante todo el tiempo que duró la investigación y que permite dar respuesta a la pregunta de investigación, es decir, una escuela básica municipal en proceso de revisión de su reglamento de convivencia escolar.

Es una investigación enmarcada dentro de una metodología cualitativa, que permite estudiar realidades complejas a través de métodos abiertos y considerar por una parte elementos contextuales que son parte del fenómeno y por otra, la subjetividad tanto de los participantes como del investigador (Flick, 2004).

\subsection{PARTICIPANTES}

Se consideró para este estudio un establecimiento educacional municipal de la comuna de Curicó. Dicha escuela contaba con un cuerpo docente formado por un director, una jefa de 
UTP, 46 profesores básicos, 6 educadores diferenciales, un inspector general, 12 asistentes de aula (de prekinder a $4^{\circ}$ básico), 4 auxiliares de aseo, 1 secretaria y 2 inspectores de patio. Su matrícula era de 536 alumnos, distribuidos en dos cursos por nivel de prekinder a $8^{\circ}$ básico. La infraestructura era de buena calidad, que consistía un edificio relativamente nuevo de 3 pisos, con salas de clases amplias y bien iluminadas, dos laboratorios de computación, biblioteca, comedor, sala de arte, sala de ciencias, aula recursos del proyecto de integración, 3 oficinas, patio y multicancha techada.

En esta escuela se estaba ejecutando un proyecto psicosocial de prevención de violencia escolar, que contaba con una psicóloga educacional que cumplía el rol de coordinadora técnica, una dupla psicosocial y un profesor de educación física. Para diseñar esta intervención en su conjunto, se utilizaron estrategias y acciones del modelo integrado de convivencia escolar (Torrego, 2006) y de la política nacional de convivencia escolar (Ministerio de Educación, Chile, 2011).

El reglamento de convivencia escolar existente en la escuela al inicio de la intervención fue elaborado en base a una asesoría externa realizada por una ATE, contratada por la Dirección comunal de educación. Dicho reglamento iniciaba con un marco legal, continuaba con una definición de conceptos, posteriormente enumeraba una serie de derechos y deberes de cada actor, para concluir con protocolos de actuación frente a situaciones de bullying, maltrato infantil y abuso sexual. Este reglamento presentaba importantes similitudes con aquellos elaborados para otras escuelas de la comuna.

\subsection{PROCEDIMIENTO}

La puesta en marcha del proceso de construcción de normas de manera participativa se realizó a través de reuniones con representantes de todos los estamentos de la escuela, así como también, reuniones de discusión por estamentos. Para esto, se utilizaron elementos del método investigación-acción, el cual se ocupa de problemas que no puede resolver la investigación pura, ya que propone un proceso en espiral donde los actores son protagonistas en la búsqueda y puesta en práctica de soluciones a los problemas identificados y donde el conocimiento se construye por medio de la práctica (Boggino y Rosekrans, 2004; Sandín, 2003). Según este método, la figura del mediador/asesor puede cumplir diferentes roles, entre ellos asistir a los docentes en el diseño y planificación del proceso; estimular, provocar y facilitar la reflexión sobre la práctica; y ayudar en la recolección de los datos y en su procesamiento, facilitando el análisis y sistematización de los resultados (Boggino y Rosekrans, 2004).

La primera reunión tuvo por objetivo involucrar a cada actor para que tome un rol activo en el proceso participativo, especialmente en las discusiones por estamento. Luego, se realizaron sesiones de discusión por estamento: dos con profesores, dos con apoderados, una con asistentes de la educación y una con estudiantes. En ellas, se utilizaron diversas metodologías que tenían por objetivo facilitar el diálogo y el análisis crítico respecto de la situación actual y cómo acercarse a una situación ideal respecto del establecimiento de normas de convivencia en la escuela. Para concluir el proceso, se planificó una última reunión donde se sintetizarían los resultados de las discusiones por estamento y se redactaría el reglamento final, la cual no se pudo realizar por problemas de agenda de director y profesores, ya que coincidió con el fin del año escolar. Por este motivo, la mediadora realizó la redacción del nuevo reglamento en base a los resultados e interpretaciones de las discusiones por estamento y del análisis del reglamento inicial. 
Este nuevo documento se estructuró de modo que iniciaba con tres valores centrales a partir de los cuales se desprenden de cuatro a seis normas de cada uno. Se decidió dejar fuera aquellos elementos relacionados con aspectos más administrativos, como los horarios o el uso de uniforme, que corresponden al reglamento interno, y se mantuvo los protocolos de actuación frente a situaciones específicas.

\subsection{RECOLECCIÓN DE DATOS}

Los datos fueron recogidos tanto del registro de campo llevado por la mediadora durante el proceso, como de focus group con profesores y asistentes de la educación una vez finalizado este. El registro de campo fue elaborado por la mediadora durante todo el proceso, desde el ingreso al establecimiento hasta la presentación del reglamento. Inicialmente este registro se realizó por escrito posterior a cada sesión, sin embargo, por la gran cantidad de información obtenida y la complejidad de las temáticas abordadas, se cambió a registro de audio, el que fue grabado inmediatamente después de realizada cada actividad y posteriormente transcrito.

Los focus group, tenían por objetivo conocer las percepciones de los actores sobre el proceso del cual ellos fueron parte. En el focus group de profesores, participaron ocho docentes de educación básica y se realizó en la sala de profesores en un tiempo destinado a labores administrativas, mientras que en el de asistentes de la educación, hubo sólo dos personas, ya que no fue posible realizar la actividad el día pactado y durante la nueva fecha el resto de estos funcionarios estaba haciendo el inventario de materiales. La participación tanto de profesores como de asistentes de la educación fue voluntaria, para lo cual, cada uno de ellos firmó un consentimiento informado. Cada focus group fue grabado en audio, lo cual se explicita tanto en el consentimiento informado como de manera verbal antes de comenzar con las preguntas, y posteriormente transcrito para su análisis.

\subsection{ANÁLISIS DE DATOS}

Para el análisis se utilizó la Teoría Fundamentada, en el cual los resultados provienen de los datos y no de teorías previamente conocidas por el investigador. Permite conocer en profundidad el desarrollo del problema estudiado, así como también explorar áreas sustantivas sobre las cuales se conoce poco, generando hallazgos que no son posibles de concebir por medio de procedimientos estadísticos u otros medios de cuantificación, utilizando la interpretación como medio para obtener los resultados (Strauss y Corbin, 2002).

Se realizó primero una codificación abierta, proceso por medio del cual los datos se descomponen en partes, siendo examinados minuciosamente y comparados entre sí en busca de similitudes y diferencias. A partir de esto, se identificaron los conceptos, con sus propiedades y dimensiones, generando agrupamiento de estos en categorías y subcategorías. Posteriormente se realizó la codificación axial, donde se establecieron relaciones entre estas categorías y subcategorías, identificando y relacionando las condiciones causales, las condiciones intervinientes, las interacciones entre elementos, junto a las consecuencias y el contexto del fenómeno estudiado (Strauss y Corbin, 2002). 


\section{RESULTADOS}

A continuación, se presentan los resultados obtenidos a partir del análisis del focus group con profesores, el focus group con asistentes de la educación y las notas de campo registradas durante todo el proceso. En primer lugar, se realizó la codificación abierta, donde se identificó las fases o momentos que efectivamente se llevaron a cabo durante el desarrollo del proceso participativo de construcción de normas de la escuela estudiada y posteriormente, se describió facilitadores, obstaculizadores y beneficios que se registraron durante el proceso. Por último, se relacionan las categorías en una codificación axial.

\subsection{DESARROLLO DEL PROCESO}

Para responder a la pregunta de investigación, respecto de cómo es el desarrollo de un proceso participativo mediado de construcción de normas de convivencia escolar, se identificaron las siguientes etapas, que se describen a continuación en orden cronológico:

\subsubsection{Toma de decisiones}

Si bien, el objetivo de este proceso es que las normas fueran construidas de manera participativa, la decisión inicial de ponerlo en marcha no lo es. Previo al proceso, existe una decisión de a quién convocar, cuándo, de qué modo, y anterior a eso, quién decide que se haga esa convocatoria. En el caso de la escuela estudiada, es el encargado de convivencia, junto a la mediadora quienes deciden convocar representantes de los distintos estamentos para debatir sobre la reformulación del reglamento. Esta decisión, pasa por el visto bueno del director, quien autoriza y se involucra en la primera reunión. Esta etapa, si bien ocupa el primer lugar en orden cronológico, fue la última en ser identificada debido a que cuando se planificó el proceso se asumió que el inicio era la primera reunión y no la decisión de iniciar el proceso, "Conversando en el recreo con [encargado de convivencia], él me planteó la posibilidad de invitar alumnos (a la reunión), yo le planteé la posibilidad de invitar apoderados. Lo discutimos." (Nota de campo).

\subsubsection{Información (sobre el proceso)}

Una vez que se toma la decisión de iniciar este proceso, se procede a informar a los diferentes miembros de la comunidad educativa, que se debatirá en torno a construir normas de convivencia compartidas por todos. La fase de información en el caso estudiado fue bastante sencilla, ya que en los actores participantes existió un alto nivel de motivación para discutir los temas propuestos, por lo tanto, fue una etapa breve, asociada básicamente a convocar y reunir a los participantes y posteriormente explicar de qué se trata el proceso, "Inicia don [encargado de convivencia] da la bienvenida, señala la importancia de que hay a representantes de todos los estamentos (...) luego me da la palabra." (Nota de campo).

\subsubsection{Problematización}

Esta etapa tiene por objetivo dar cuenta del malestar de los participantes con la situación actual, lo cual debiera movilizarlos posteriormente a generar propuestas. Aquí, la presencia 
de la mediadora fue fundamental, ya que lidera y facilita la participación, generando preguntas abiertas que permiten el análisis de las múltiples dimensiones del problema (en este caso, el reglamento) desde aspectos más estéticos como su extensión y la forma en que está redactado, hasta temas más de fondo, como lo son el contenido de las normas, la interpretación que se hace de ellas, las personas que las aplican, el nivel de acuerdo actual con estas, etc.

De este modo, aparecen problemas como que el reglamento era muy extenso y está redactado con un lenguaje complejo, lo que hacía difícil su comprensión y socialización hacia todos los actores.

Y porque es muy largo, muy extenso, porque hay que ir explicando uno por uno, porque también está escrito de una manera que es incomprensible (Profesora).

Es que hay cosas en el reglamento interno que uno no entiende (Asistente).

La amplia extensión del reglamento tiene además el inconveniente de que las personas no se interesan por leerlo, lo que genera desconocimiento en casi la totalidad de los actores respecto de las normas de convivencia escolar.

O está en la agenda y no se da el trabajo el apoderado ni siquiera de leerlo y se encuentra de repente cuando ya los niños han infringido alguna norma o han tenido alguna dificultad, uno les dice "lea la agenda" y ellos recién ahí se toman el tiempo. (Profesora)

les mostré el reglamento de la agenda y nadie lo había leído, coincide con el resto. Sí, definitivamente nadie lee este reglamento. (Nota de campo)

Por otra parte, se observó entre los niños una actitud negativa generalizada hacia las normas, lo que genera que la mayoría de ellos consideren que las normas existentes no tienen utilidad y que su transgresión, es una posibilidad válida para ellos.

y hubo varios que me dijeron "me salto las normas cuando: siempre", "las normas me ayudan cuando: nunca”, o sea, la mayoría, salvo pocas excepciones, pero lamentablemente hubo muchos niños que ven las normas como algo muy, muy negativo. (Nota de campo)

\subsubsection{Debate}

Lo más relevante de esta etapa es que una vez que se identificaron los problemas, se espera que aparezcan propuestas o ideas de solución. Una de ellas fue la necesidad de consenso, en especial en el estamento profesores, quienes valoran que se hayan generado discusiones sobre el tema, ya que les ayudó a generar acuerdos para el abordaje de problemas de convivencia.

en algunos problemas de algunos niños que podemos dar todos soluciones y de repente nos sentimos como encerrados ¿qué podemos hacer con él? Pero a lo mejor otro, otro 
colega tiene la forma de "a ver mira, haz esto, haz esto otro, hagamos esto, hagamos esto otro". (Profesora)

Otro punto importante que aparece es la necesidad de trabajar con las familias, ya que el estamento "apoderados" resulta especialmente conflictivo dentro de la escuela estudiada, y los debates fueron de gran utilidad para abrirse a nuevas posibilidades y plantear propuestas.

hacer, quizás una reunión con los alumnos, por ejemplo, apoderados y alumnos en la misma reunión, y de repente igual es bueno, porque muchas veces el apoderado llega a la casa y yo creo que son pocos los que comentan lo que se habló, algunos llegan quizás a dormir, no sé. (Profesor)

Por último, el debate permite que los actores sientan el reconocimiento por sus aportes dentro de la escuela, lo que valoriza su rol como actores, más allá del estamento al cual corresponden, "les pregunté que qué les había parecido la actividad, todos (los niños) reconocieron que era una actividad buena, porque les habían preguntado la opinión, o sea, valoraron mucho que se les preguntara la opinión" (Nota de campo).

\subsubsection{Interpretación}

Esta etapa es desarrollada por la mediadora en conjunto con el encargado de convivencia y el equipo de intervención de manera paralela a las fases de problematización y debate. Culmina con la redacción del nuevo reglamento participativo que se lleva a cabo de forma posterior a las dos etapas mencionadas. Es una etapa muy delicada, que requiere conocimientos sobre la cultura escolar, mucho trabajo de campo, observación y análisis de la escuela, análisis en profundidad de las discusiones entre participantes, empatía, pragmatismo, intención de generar consenso y visualización de las consecuencias que estas interpretaciones pueden traer a la escuela.

La fase de interpretación implica explorar varias las alternativas antes de generar el producto final, ya que consiste justamente en dar estructura a los contenidos producidos durante las discusiones, que por definición no la tienen.

Yo le planteé que como, cómo armamos esto finalmente, cómo lo ordenamos. Porque una de mis opciones era... eh, poder hacer una especie de decálogo, la primera idea, cuando recién partimos era hacer una especie de decálogo de la convivencia, que ese fuera el reglamento. Después de eso me quedé pensando en el tema de las sanciones, porque las sanciones es el tema más complejo. Y... después pensé que tal vez no era necesario este decálogo porque a lo mejor, era mejor esto de separar, así como en la ley están las faltas y los delitos, acá también, eh, separar o clasificar las cosas que se pueden resolver entre pares o al interior del curso, de aquellos temas que son más graves. Por otra parte, tenemos una reformulación de los valores del PEI, que en este momento los valores son "disciplina y esfuerzo" y yo lo cambiaría por "respeto y comunicación". Y poner los valores institucionales en el primer, en eh, como arribita, como que todo el mundo los conozca y a partir de ahí establecer otra, otras cosas más específicas. Ya, así que ahí estamos medios perdidos respecto de cómo vamos a... 
cómo vamos a redactar este documento. Eso, complicado. Complicado, me siento un poco... perdida. (Nota de campo)

Finalmente, se decidió generar un reglamento que contenía tres valores centrales: respeto, comunicación y orden. Los dos primeros, aparecieron explícitamente en las discusiones de los diferentes actores, mientras que el último se extrajo del reglamento anterior observándose también de manera implícita en las discusiones. A partir de estos valores, se generan normas vinculadas a ellos, redactadas de manera breve y utilizando un lenguaje sencillo.

La fase de interpretación concluye al momento de hacer la devolución del nuevo reglamento a los participantes (por motivos de tiempo sólo se realizó a algunos profesores y asistentes), quienes declararon sentirse completamente identificados con el nuevo reglamento construido, junto con no percibir riesgos de que la mediadora hubiera realizado una interpretación errada.

\section{Yo le hubiese dicho (...) ¿Y qué pasó con lo que hablamos? (Asistente)}

Que tú presentes el proyecto final, nosotros te vamos a decir, "mira, sabes que creemos que", se supone, en la práctica que deberíamos hacerlo "esto, creemos que no corresponde a lo que el colegio requiere". (Profesora)

\subsubsection{Información (para la toma de decisiones)}

Esta es una etapa bastante sencilla pero sumamente importante para que, tanto el proceso participativo como las normas construidas a partir de él, trasciendan al interior de la institución. Es el momento en que toda la comunidad educativa toma conocimiento del documento final que contiene el reglamento para aprobarlo o rechazarlo. Es importante que la comunidad esté consciente de que las normas fueron construidas por todos los actores, porque de lo contrario, se corre el riesgo de mantener la lógica jerárquica del reglamento impuesto que existía previo al proceso, al señalar que este debe ser "socializado", "O sea hay que sociabilizar todas esas normas con los apoderados, con los profesores y con los asistentes." (Profesora).

\subsubsection{Toma de decisiones, seguimiento y evaluación}

Para finalizar este proceso, se hace necesario realizar, por una parte, la toma de decisiones que implique la formalidad necesaria para la validación del nuevo reglamento a nivel institucional, así como también el seguimiento para verificar empíricamente que el nuevo reglamento cumple con los objetivos planteados y, finalmente, la evaluación que busca analizar la efectividad del reglamento construido y realizar ajustes que sirvan para mejorar en el futuro. Estas fases son hipotéticas, ya que no se llevaron a la práctica por motivos de tiempo.

\subsection{FACILITADORES DEL PROCESO}

Junto a las etapas descritas anteriormente, también es importante destacar aquellos factores que facilitaron el proceso de construcción de normas en el colegio estudiado, donde destacan la figura del mediador y la construcción de confianza. 


\subsubsection{Importancia del mediador}

Los propios actores definen el rol del mediador como fundamental y reconocen que, sin la presencia de este, el proceso de revisión de normas de convivencia de manera participativa hubiera sido muy complejo o no se hubiera podido realizar, "Porque tiene que haber alguien que dirija, o sea, alguien que dé ideas, y quién más que usted que es psicóloga." (Asistente).

Así también reconocen la necesidad de que este mediador sea externo, de manera de garantizar una visión más neutral al interior de la institución que pueda reflejar las opiniones de los diferentes estamentos: "Que no esté relacionado con el tema, (...) yo creo que es mucho más conveniente que sea una persona externa al colegio." (Profesora).

\subsubsection{Construir confianza}

Uno de los mayores desafíos durante el proceso fue construir confianza entre los actores. Fue posible observar, en base a diferentes opiniones durante el proceso, que muchas personas son reacias a participar porque no confían en los resultados que se puedan generar, produciendo a su vez, malos resultados. Se crea así una especie de círculo vicioso entre falta de confianza y falta de participación.

Justamente la participación de todos los actores al inicio del proceso, durante la primera reunión, ayudó a que aumentara la confianza en que lo que se estaba haciendo, ya que generó la percepción de tener el respaldo de todos en la escuela. Esto además ayuda a que los otros actores aumenten sus expectativas respecto del proceso y se motiven más por participar, "Sólo iba a escuchar. Pero a medida que fue pasando la reunión vi que la cosa era muy interesante, muy interesante y había involucrada mucha gente que no pensé que estaría ahí." (Asistente).

\subsection{OBSTACULIZADORES DEL PROCESO}

Así como existieron factores que facilitaron el proceso, también hubo otros que lo hicieron más difíciles. A continuación, se describen para tenerlos en cuenta e intentar minimizar sus efectos en caso de realizar este proceso en oportunidades futuras.

\subsubsection{Poco tiempo}

Al momento de preguntar explícitamente a los diferentes actores respecto de qué obstaculizadores perciben respecto de la participación, lo más mencionado son las dificultades de tiempo en diferentes niveles. Por ejemplo, el tiempo limitado para concejos de curso y reuniones de apoderados.

Yo siento que una hora de reunión de apoderados se hace muy poco. (Profesora)

45 minutos es muy poco tiempo, sobre todo cuando se hacen dinámicas (con los niños), en mi curso, las dinámicas quedaban a la mitad. (Profesora)

Así también los profesores, especialmente aquellos que desempeñan el rol de profesores jefes señalan que no tienen tiempo para abordar problemas de convivencia ni para trabajo 
colaborativo con otros docentes, lo que muchas veces genera que el abordaje de problemas de convivencia se haga interrumpiendo las horas de clases.

$Y$ el poco tiempo que tenemos todos, porque a veces yo voy a tener un rato libre $y$ quizás yo me voy a poder acercar a X colega o al inspector o a quien sea, pero el resto de mis colegas no lo va a poder hacer, o yo quizás tuve un problema con un curso, pero no tengo el tiempo o mi colega no tiene el tiempo para que lo abordemos. Muchas veces pasa que uno tiene algún problema con un curso y tiene que salir de la hora de clase para solucionar el problema, porque no hay más tiempo. (Profesora)

\subsubsection{Sobrecarga de tareas y prioridades}

Otra dificultad observada es que debido a las múltiples exigencias que tiene el sistema escolar, las temáticas de convivencia escolar no siempre son una prioridad para los actores. Reconocen que el sistema educacional en general, da prioridad a aquellas asignaturas medidas en pruebas estandarizadas y su entrenamiento, en detrimento de asignaturas que tienen como objetivo trabajar lo valórico.

Pero yo creo que un alumno responsable, un alumno que tú dices, valores firmes, que se le trabajaran los valores, eh, rendiría mejor en una prueba. Estamos muy mal enfocados en ese tema, porque pasamos contenidos, porque, bueno, el SIMCE lo que pide es eso, contenidos. (Profesora)

Así como en los profesores, el énfasis por los contenidos les quita espacio para lo valórico y formativo, en el caso de los asistentes, su participación se ve afectada por dar mayor relevancia a sus tareas cotidianas, no siendo prioridad para ellos las discusiones en torno a la convivencia escolar, por considerarse a sí mismos actores "poco relevantes".

Yo creo que por eso (no participan), por el tipo de presión que tienen, les exige, y entonces al tomar una decisión de que bueno, lo hago o no lo hago, si no lo hago me puede llegar por otro lado. Entonces, por eso se van. (Asistente)

\subsubsection{Experiencias previas de frustración}

Otro obstaculizador importante son las experiencias de frustración que diferentes actores habían experimentado previamente, y que de alguna manera condicionan y limitan su disposición a participar de las actividades actuales.

Ahí va a quedar y no va a haber solución. Lo dicen muchas acá. Entonces yo creo que por eso no participan. (Asistente)

Porque siempre las cosas aquí en educación llegan y se van así. Así como llegaron se van. Y nada va con evaluación, o sea, no se evalúa al final, no tiene un seguimiento que eso es lo importante, el seguimiento, porque no tiene sentido hacer una cosa y después terminar inmediatamente. Y eso es lo que pasa con la mayoría de los programas. (Profesora) 


\subsubsection{Conflictos intraestamentales}

Otro obstaculizador importante para generar diálogo y consenso son los conflictos intraestamentales. Son especialmente evidentes los que ocurren entre apoderados, en la instancia de reunión, lo que genera que estos espacios, que ya son breves, sean tediosos, poco productivos y generen aún más experiencias de frustración.

Una apoderada me dijo que había muchos conflictos "entre" apoderados en reunión, que a ella le había pasado en su curso que uno decía una cosa y el otro se ponía a alegar y el otro alegaba también, y alegaban y al final se hacían puras peleas, pero no peleas generadas por el profesor o por el contenido, sino que peleas entre ellos. (Nota de campo)

\subsection{BENEFICIOS DEL PROCESO}

Para finalizar, se describen tres beneficios obtenidos en los miembros de la comunidad escolar, como consecuencia del proceso participativo de construcción de normas de convivencia escolar:

\subsubsection{Generar aprendizajes en los actores}

El beneficio más importante de construir participativamente normas de convivencia es que genera aprendizajes en quienes participan en él. En el caso de los niños, se observa que al reflexionar respecto de por qué son necesarias las normas, descubren que finalmente, cada una de ellas tiene un sentido que está relacionado con el rol de la escuela como espacio formativo.

Lo bueno es que al final (...) (un niño) dijo, para qué queremos recreos más largos si vamos a salir a las doce (...) o alguien que dijo "no hagamos clase" y entonces la otra niña dijo "y entonces qué vamos a aprender". Entonces yo creo que esas son cosas que hay que rescatar, porque son razonamientos importantes que hicieron y que son positivos. (Nota de campo)

Así también, en el caso de los asistentes de la educación, se puede observar claramente cómo perciben y valoran que se les haya incluido en estas instancias donde tuvieron la posibilidad de aportar y aprender, "Yo esa vez que me invitaron yo escuché todo y a mí me gustó, me gustó porque lo encontré entretenido, porque aprende uno de cada reunión, uno siempre aprende algo, algo diferente". (Asistente).

\subsubsection{Estructura del nuevo reglamento}

Uno de los beneficios que se producen al problematizar primero, debatir e interpretar después, es que el nuevo reglamento se construye de tal manera que supera los problemas iniciales detectados, para dar paso a una estructura y redacción más práctica y amigable, la cual incluye un reglamento más breve y sencillo, que resulta de fácil comprensión y más posible de llevar a la práctica. 
O sea a mí, como auxiliar me gustó, me encantó eso, que sea bien clarito. (Asistente)

Creo que está bien, está bastante sintetizado (...) está como bien preciso. Y va a ser mucho, creo que más fácil trabajar que todas las otras cosas que hemos puesto. (Profesora)

Así también, fue altamente valorado que en el nuevo reglamento estuvieran unidas las normas y los valores, ya que se entiende que estos últimos son universales, independiente de las jerarquías y roles al interior del establecimiento, y estos a su vez, dan sentido a las normas.

Yo creo que está bien que los valores estén enlazados con, con normas después (...) Entonces se entiende que hay como una lógica. (Profesora)

Habla del respeto hacia el resto, que no sólo el respeto hacia el profesor, hacia el paradocente, también respeto hacia nosotros, como nosotros tenemos respeto hacia el resto, entonces también va todo como de la mano. (Asistente)

\subsection{CODIFICACIÓN AXIAL}

Finalmente, se construyó un esquema de relaciones entre las categorías descritas (figura 1), donde el fenómeno central es el proceso participativo de construcción de normas realizado por la comunidad educativa de la escuela estudiada, ya que es el fenómeno que da respuesta a la pregunta de investigación. El contexto donde se sitúa el fenómeno estudiado es la gestión de la convivencia escolar en la escuela. La toma de decisiones inicial que, si bien es descrita en la codificación abierta como parte del desarrollo del proceso, más que una etapa de este sería una condición causal debido a que da paso a este proceso. Las interacciones identificadas fueron las etapas del proceso de construcción de normas, donde los datos arrojan una descripción detallada de las dinámicas del proceso. Dentro de las condiciones intervinientes, encontramos los facilitadores y los obstaculizadores todos los cuales están presentes e interactúan durante todas las etapas del proceso. Por último, como consecuencias encontramos los beneficios identificados, donde están la síntesis y consenso en el nuevo reglamento, los aprendizajes obtenidos por los actores participantes y la articulación de normas y valores. 
Figura 1. Codificación axial.

Contexto: Gestión de la convivencia escolar en una escuela básica municipal.

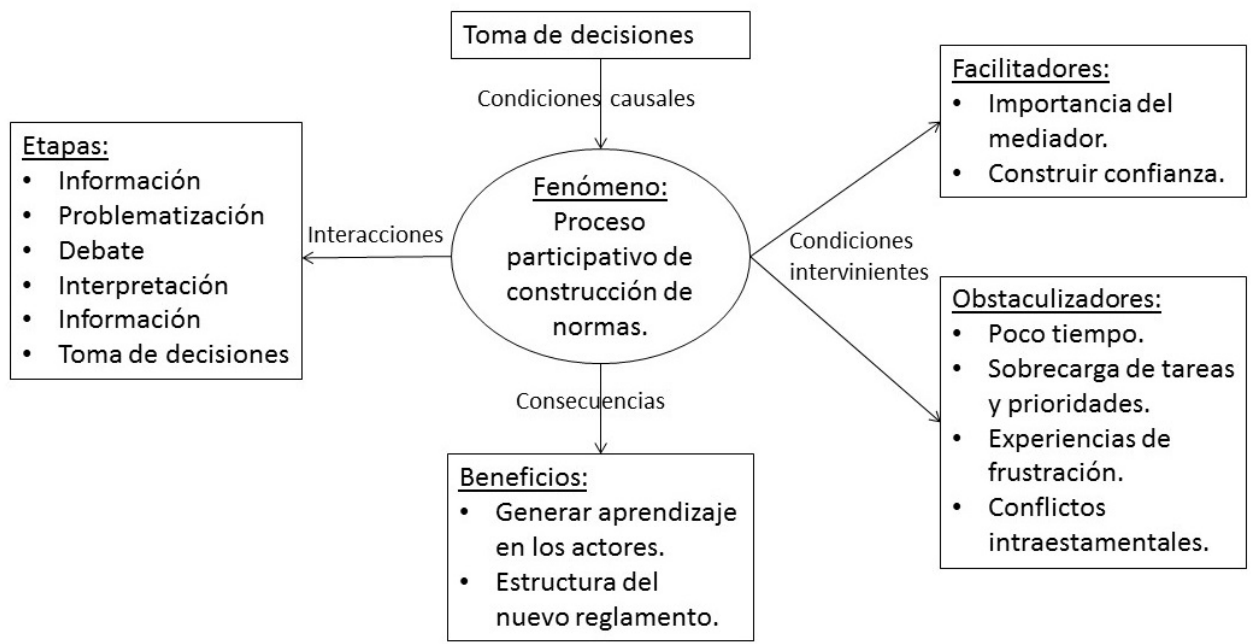

\section{DISCUSIÓN Y CONCLUSIONES}

Una vez concluido el proceso, es posible señalar que hacer partícipe a la comunidad escolar permitió a la escuela abrir un repertorio de prácticas que le pueden permitir transitar desde una visión reducida de la convivencia escolar, centrada en la disminución de la violencia por parte de los niños, para pasar a concebirla desde una visión amplia (Carbajal, 2013), donde se involucra a toda la comunidad educativa, de modo que los actores sean protagonistas en la búsqueda y puesta en práctica de la construcción y consolidación de relaciones pacíficas entre los miembros de la comunidad escolar. Lo anterior también implica revalorizar el conflicto desde un paradigma crítico (Jares, 1997), ya que en medio de la amplia diversidad de ideas y creencias arraigadas en los diferentes actores, no se puede continuar con el intento de llevar a la práctica la fantasía de escuelas libres de conflicto, sino que, por el contrario, implica reconceptualizar estos, buscando llegar a las raíces estructurales de este, generando un paso paulatino hacia relaciones sociales más justas y democráticas (Carbajal, 2013).

Lo fundamental que resultaron ser los elementos de la investigación-acción empleados dan cuenta de que su uso en un proceso como este, nos entrega valiosas herramientas metodológicas para generar prácticas transformadoras en el área de la convivencia escolar, sobre todo en aquellas escuelas que presentan abundantes problemas en esta área, debido a que posee aspectos clave como por ejemplo, parte de problemas prácticos, implica la colaboración de las personas, implica una reflexión sistemática en la acción, se realiza por las personas implicadas en la práctica que se investiga, el elemento de "formación" es esencial y se define como un espiral de cambio (Sandín, 2003), por lo que la metodología utilizada durante el proceso se considera no sólo adecuada, sino que además recomendable para abordar problemas como el que se analizó en esta investigación. 
Si analizamos qué nivel de participación que se alcanzó, es posible señalar que el proceso realizado en la escuela estudiada supera el clásico esquema de niveles de participación que incluye información, consulta, toma de decisiones y control de eficacia (Flamey et al., 1999; Gubbins y Berger, 2002; Romagnoli y Gallardo, 2007). Esta descripción pareciera ver a la participación desde una visión vertical e institucional, donde sólo se considera a la comunidad para aprobar o desaprobar una propuesta, previamente definida por otros. En cambio, en el proceso llevado a cabo durante esta tesis, el nivel de participación alcanzado es el de "construcción colectiva", es decir, se genera un producto nuevo con una lógica distinta (no predeterminada) a partir de los aportes que los diferentes actores hacen, desde su propia perspectiva respecto del fenómeno en cuestión, acercándose hacia deconstruir la cultura escolar predominante para generar un producto nuevo, en el cual la participación no es un mero instrumento metodológico, sino que es la sustancia, la materia prima para construir las pautas de relación a través de las cuales se espera alcanzar una sana convivencia.

Respecto del resultado final del proceso, se logró generar un nuevo reglamento de convivencia, que lamentablemente no fue aplicado por la escuela, manteniéndose el antiguo, con todos los problemas ya descritos por los actores. Sin embargo, no podemos decir que esta construcción de normas no tuvo ningún efecto en la comunidad educativa sólo porque en la formalidad el reglamento no fue adoptado legalmente. En este sentido, generar espacios de diálogo en la escuela es "en sí mismo" un aporte a la comunidad educativa, debido a que lo que se construye a través del diálogo entre los miembros de una comunidad, puede tener siempre un potencial resolutivo en la medida que se genere confianza, se tomen decisiones democráticas y el resultado final tenga utilidad para resolver problemas prácticos. Por lo tanto, un reglamento que sea capaz de generar buena adherencia a las normas y prevenir situaciones de violencia, puede generar que esas normas orales tengan sentido y propósito, para las comunidades educativas que las ponen en práctica, aun cuando estas no sean válidas legalmente. Por lo anterior, es tan importante al interior de las escuelas "revalorizar la cultura oral y los conocimientos extraídos de la experiencia del niño o del adulto" (Organización de Naciones Unidas para la Educación, la Ciencia y la Cultura, 1996, p. 107). La revalorización de los acuerdos orales puede llevar a un nivel de utilidad práctica, más allá de si los reglamentos escritos, con sus respectivos procedimientos burocráticos lo permiten, tal como lo relata una apoderada en el estudio sobre concejos escolares realizado por De la Fuente y Razcynski (2010) el cual señala:

Cuando un CE (concejo escolar) trabaja bien... hace una planificación, una estrategia, un seguimiento, pasa a ser resolutivo... Cuando uno se propone algo y lo logra es resolutivo... No necesitas que te digan que el CE es resolutivo, se trata de hasta qué punto puede llegar a serlo si se trabaja con un objetivo claro (p. 49).

Por esto, el real aporte de generar normas de convivencia escolar a partir de procesos de diálogo entre actores es que siempre tiene un potencial resolutivo.

El principal aporte de esta investigación es que da luces de cómo generar procesos participativos al interior de las comunidades escolares en el área de la convivencia, lo que permite superar la aparente desestructuración que genera en las escuelas la percepción de que la participación es problemática (Sús, 2005), entregando un modelo que entrega estructura y flexibilidad a la vez, ya que describe un proceso con ciertas etapas bien 
caracterizadas, que son perfectamente replicables en otras escuelas, y a la vez permite flexibilizar en los métodos, adecuándose a las especificidades contextuales de cada escuela. Junto con esto, se describen facilitadores y obstaculizadores a tener en cuenta a modo de despejar dudas que inevitablemente van surgiendo durante el proceso. En este sentido, se ha logrado analizar y describir un proceso participativo de convivencia escolar, que una vez concluido permite promover e incentivar la participación en el área de convivencia escolar en todas las escuelas.

También es posible señalar que este estudio constituye un aporte al desarrollo de la psicología educacional, tanto desde la investigación empírica como desde la práctica profesional, ya que abre posibilidades de posicionamiento a los psicólogos educacionales como mediadores de participación, apoyando a que cada día más comunidades educativas implementen prácticas participativas en la gestión de la convivencia, no sólo por los beneficios en su mejora, sino que también porque constituye un desafío para generar aprendizajes en las áreas valóricas y de formación ciudadana.

Dentro de los desarrollos futuros que se pueden generar a partir de esta investigación, podemos destacar, desde la práctica, replicar el diseño con un mejor ajuste a los tiempos de la institución, de modo de poder realizar seguimiento, evaluación y rediseño, esto último, para destacar el carácter de espiral que tiene un proceso que se va mejorando en la medida que se vuelve a dialogar una y otra vez en relación a las normas ya creadas. Así también, se puede estudiar a ciertos actores específicos, de tal modo de conocer las particularidades que cada uno pueda tener dentro del desarrollo del proceso y cómo influyen las jerarquías y la micropolítica en cada actor específico. Por último, indagar en qué medida la tradición oral logra imponerse por sobre lo que está escrito o si esto último prevalece sobre lo primero.

El último y más importante desafío de llevar a cabo esta investigación es lograr trascender al seno de las comunidades educativas que hoy en día aún se ven disminuidas por prácticas autoritarias y en exceso jerárquicas que brindan pocos espacios de desarrollo a sus miembros. Instaurar cambios en la cultura escolar instalando procesos más participativos y democráticos que se traduzcan también en una formación más integral de los niños y de toda la comunidad educativa es un objetivo que necesariamente implica la convergencia de la investigación empírica, las políticas públicas y las prácticas cotidianas al interior de las escuelas, donde la presente investigación intentó entregar herramientas metodológicas que permitan avanzar en esa dirección.

\section{REFERENCIAS BIBLIOGRÁFICAS}

Arón, A. y Milicic, N. (2000). Climas sociales tóxicos y climas sociales nutritivos para el desarrollo personal en el contexto escolar. Psykhé, 9(2), 117-123.

Ball, S. (1994). La micropolítica de la escuela. Hacia una teoría de la organización escolar. Paidós.

Bardisa, T. (1997). Teoría y práctica de la micropolítica en las organizaciones escolares. Revista Iberoamericana de educación, 15, 13-52.

Boggino, N. y Rosakrans, K. (2004) Investigación-acción: reflexión crítica sobre la práctica educativa. Orientaciones prácticas y experiencias. Homo Sapiens.

Bugueño, X. y Mena, I. (2008). El sentido del reglamento de convivencia: lógicas y sugerencias para su construcción. Documento Valoras UC. http://valoras.uc.cl/images/centro-recursos/ docentes/GestionLiderazgoInstrumentosConvEscolar/Fichas/El-sentido-del-reglamento-deconvivencia.pdf 
Carbajal, P. (2013). Convivencia democrática en las escuelas. Apuntes para una reconceptualización. Revista Iberoamericana de evaluación educativa, 6(2), 13-35.

Casas, L., Ahumada, C., Ramos, L. y Guajardo, A. (2008). La convivencia escolar, componente indispensable del derecho a la educación (Estudio de Reglamentos Escolares). Serie Infancia y Adolescencia UNICEF, 11, 7-24.

De la Fuente, L y Raczynski, D. (2010). Estudio de evaluación de la situación de los concejos escolares. Informe Final. Asesorías para el desarrollo. http://www.asesoriasparaeldesarrollo.cl/ informes-y-estudios/

Flamey, G., Gubbins, V. y Morales, F. (1999). Los centros de padres y apoderados: nuevos actores en el control de la gestión escolar. Cuadernos de Educación, 4, 1-37. http://biblioteca.uahurtado.cl/ UJAH/856/txtcompleto/txt96897.pdf

Flick, U. (2004). Introducción a la investigación cualitativa. Morata.

Gubbins, V. \& Berger, C. (2002). Hacia una alianza efectiva entre familias y escuelas. Persona y sociedad, 16(3), 71-86.

Hart, R. (1993). La participación de los niños. De la participación simbólica a la participación auténtica. Ensayos Innocenti, UNICEF, 4. https://www.unicef-irc.org/publications/pdf/ie_ participation_spa.pdf

Jares, X. (1997). El lugar del conflicto en la organización escolar. Revista Iberoamericana de Educación, 15, 53-73.

(2006). Conflicto y convivencia en los centros educativos de secundaria. Revista de Educación, 339, 467-491.

Ley sobre violencia escolar 20.536/2011, 17 de septiembre. Biblioteca del Congreso Nacional. http:// www.mineduc.cl/usuarios/convivencia_escolar/doc/201109221119290.ley_violencia_escolar. pdf

Litichever, L. y Núñez, P. (2005). Acerca de lo justo, lo legal y lo legítimo. Cultura política en la escuela media. Última Década, 23, 103-130.

Mena, I., Jauregui, P. y Moreno, A. (2011). Cada quien pone su parte. Conflictos en la escuela. Editorial SM.

Ministerio de Educación, Chile. (2011). Política nacional de convivencia escolar. http://www.mineduc. cl/usuarios/convivencia_escolar/doc/201203262303500.PoliticadeConvivenciaEscolar.pdf

. (2014). Otros indicadores de calidad educativa. http://sustentabilidad.umce.cl/wp-content/ uploads/2016/08/Indicadores-de-calidad_OBT-1.pdf

. (2015). Política nacional de convivencia escolar 2015-2018. http://www.mineduc.cl/wpcontent/uploads/sites/19/2015/12/politica-noviembre-definitiva.pdf

. (2016). Orientaciones para la elaboración del plan de formación ciudadana. http:// www.mineduc.cl/wp-content/uploads/sites/19/2016/04/DEG-OrientacionesPFC-intervenibleAReader_FINAL.pdf

Mundaca, R. y Flores, C. (2014). Derechos del niño, participación infantil y formación ciudadana desde espacios educativos no formales: la experiencia del Consejo Consultivo de Niños, Niñas y Adolescentes de la comuna de Coquimbo, Chile. Temas de educación, 20(1), 123-141.

Organización de Naciones Unidas para la Educación, la Ciencia y la Cultura. (1996). La educación encierra un tesoro. Santillana.

Ortega, R. (1998). La Convivencia Escolar: qué es y cómo abordarla. Consejería de Educación y Ciencia. Junta de Andalucía. España.

Ortega, R., Del Rey, R. y Casas, J. (2013). La convivencia escolar: clave en la predicción del bullying. Revista Iberoamericana de evaluación educativa, 6(2), 91-102.

Rivera, M. y Milicic, N. (2006). Alianza familia-escuela: percepciones, creencias, expectativas y aspiraciones de padres y profesores de enseñanza general básica. Psykhé, 15(1), 119-135.

Romagnoli, C. y Gallardo, G. (2007). Alianza efectiva familia escuela: para promover el desarrollo intelectual, emocional, social y ético de los estudiantes. Documento Valoras UC. http://valoras. 
uc.cl/images/centro-recursos/familias/ValoresEticaYDesarrolloSocioemocional/ Documentos/ Alianza-Efectiva-Familia-Escuela_Para-promover.pdf

Ruiz, A. y Chaux, E. (2005). La formación de competencias ciudadanas. Ascofade.

Sandín, M. (2003). Investigación cualitativa en educación. Fundamentos y tradiciones. Mc Graw Hill.

Stake, R. (1994). Investigación con estudio de casos. Morata.

Strauss, A. y Corbin, J. (2002). Bases de la investigación cualitativa. Técnicas y procedimientos para desarrollar la teoría fundamentada. Universidad de Antioquía

Sús, M. (2005). Convivencia o disciplina ¿qué está pasando en la escuela? Revista Mexicana e investigación educativa, 27(10), 983-1004.

Torrego, J. (2006). Modelo integrado de mejora de la convivencia. Estrategias de mediación y tratamiento de conflictos. Graó.

Valdés, A. y Urías, M. (2011). Creencias de padres y madres acerca de la participación en la educación de sus hijos. Perfiles Educativos, 33(134), 99-114. 
